those who withdrew, redo the intention-to-treat analysis and calculate dichotomous weight change outcomes. This, however, would still not resolve the basic problem with regard to quality in individual studies. Well-designed, large-scale pragmatic trials with longer periods of follow-up are needed before undertaking further review in this area, an implication which has been acknowledged by the authors.

1 Álvarez-Jiménez M, Hetrick SE, González-Blanch C, Gleeson JF, McGorry PD. Non-pharmacological management of antipsychotic-induced weight gain: systematic review and meta-analysis of randomised controlled trials. Br J Psychiatry 2008; 193: 101-7.

2 Hollis S, Campbell F. What is meant by intention to treat analysis? Survey of published randomised controlled trials. BMJ 1999; 319: 670-4.

Dimple George, Academic CT3, Wolfson Research Institute, Durham University Queen's Campus, Stockton-on-Tees, UK. Email: georgedimple@hotmail.com; J. G. Reilly, Mona-Lisa Kwentoh Tees, Esk and Wear Valleys NHS Foundation Trust and School for Medicine and Health, Durham Univeristy Queen's Campus, Stocktonon-Tees, UK

doi: 10.1192/bjp.194.1.88a

Authors' reply: We would like to thank George et al for their comments. However, we believe that some clarification is needed regarding the outcomes and procedures of our meta-analysis.

First, we agree that percentage of weight gain is a more appropriate measure to assess weight gain compared with body weight change. In fact, somewhere else we have pointed to 'the importance of reporting percentage of weight gain, as absolute body weight changes may be deceptive, concealing the real extent of this side effect on those who experience weight gain. ${ }^{1}$ To put it more simply, research shows that up to $80 \%$ of individuals being treated with antipsychotics suffer significant gain in body weight. As a result, patients taking antipsychotics are more likely to gain $20 \mathrm{~kg}$ than they are to lose $20 \mathrm{~kg}$. Indeed, weight-management interventions do not usually produce weight loss but they attenuate antipsychotic-induced weight gain. ${ }^{2}$ For these reasons, data on weight change is unlikely to overestimate the effectiveness of weight management interventions as George et al contend. To illustrate this further, in a previous randomised controlled trial (RCT) of weight-management interventions we assessed the proportion of patients that gained more than $7 \%$ of their baseline body weight. Patients in the control group gained $6.9 \mathrm{~kg}$ compared with $3.9 \mathrm{~kg}$ in the intervention group. These absolute gains were translated into $78.8 \%$ in the control group increasing their baseline weight by more than $7 \% v .39 .9 \%$ in the intervention group. ${ }^{3}$

George et al also commented on the quality of the included trials as a potential threat to the reliability of the results. First, it should be pointed out that only RCTs were included - in three of them we were able to pool relevant data with the help of the authors. Second, we performed several sensitivity analyses to determine the robustness of our findings to the exclusion of low-quality trials and exclusion of small trials. ${ }^{4}$ The exclusion of these studies affected the overall effect size and confidence intervals only marginally. Importantly, there was notable consistency across all study estimates, which was reflected in the robustness of the findings across analytic methods. Thus, our findings are unlikely to be biased by these issues.

After examining all the available evidence, it is now possible to conclude that large-scale pragmatic trials with longer follow-up are needed to make further progress in this area as George et al state.

1 Álvarez-Jiménez M, González-Blanch C, Crespo-Facorro B, Hetrick S, Rodríguez-Sánchez JM, Pérez-Iglesias R, et al. Antipsychotic-induced weight gain in chronic and first-episode psychotic disorders: a systematic critical reappraisal. CNS Drugs 2008; 22: 547-62.

2 Álvarez-Jiménez M, Hetrick SE, González-Blanch C, Gleeson JF, McGorry PD. Non-pharmacological management of antipsychotic-induced weight gain: systematic review and meta-analysis of randomised controlled trials. Br J Psychiatry 2008; 193: 101-7.

3 Álvarez-Jiménez M, González-Blanch C, Vázquez-Barquero JL, Pérez-lglesias R, Martínez-García O, Pérez-Pardal T, et al. Attenuation of antipsychoticinduced weight gain with early behavioral intervention in drug-naive firstepisode psychosis patients. A randomized controlled trial. J Clin Psychiatry 2006; 67: 1253-60.

4 Egger M, Smith GD, Phillips AN. Meta-analysis: principles and procedures. BMJ 1997; 315: 1533-7.

Mario Álvarez-Jiménez, ORYGEN Research Centre, University of Melbourne, Australia, and University Hospital 'Marqués de Valdecilla', Department of Psychiatry, University of Cantabria School of Medicine, Santander, Spain. Email:

University of Cantabila School of Medicine, Santander, Spain. Email: malvarez@unimelb.edu.au; Sarah E. Hetrick, ORYGEN Research Centre, University of Melbourne, Australia; César González-Blanch, University Hospital 'Marqués de Valdecilla', Department of Psychiatry, University of Cantabria School of Medicine, Santander, Spain; John F. Gleeson, ORYGEN Research Centre, and Department of Psychiatry, University of Melbourne, and NorthWestern Mental Health Programme, Melbourne, Australia; Patrick D. McGorry, ORYGEN Research Centre, University of Melbourne, Australia

doi: $10.1192 / \mathrm{bjp} .194 .1 .89$

\section{Aetiological significance of middle-ear disease in schizophrenia}

We read the study by Mason $e a^{1}$ with great interest. The authors conclude that there is an association between middle-ear disease and schizophrenia which may have aetiological significance. However, the authors have based their conclusions on a case-control study, which is susceptible to biases and effects of confounding factors; we would like to raise concerns about these conclusions.

First, we would like to highlight the strong possibility of selection bias as this study design is particularly prone to it. In this case, at the sample selection stage, no precautions were taken to ensure that the person selecting the patients was masked to the study hypothesis. This could lead to bias towards selecting patients with middle-ear disease and schizophrenia.

Case-control studies are more susceptible to bias and confounding factors than are cohort studies. In order to establish the association, it is recommended that we should have an odds ratio $>4,{ }^{2}$ because the higher the odds ratio, the stronger the association. However, Mason et al have concluded about the association when the odds ratio is $<4$, which could be as a result of bias alone. This raises strong doubts about the validity of the authors' conclusions.

We would request that the authors clarify these issues.

1 Mason P, Rimmer M, Richman A, Garg G, Johnson J, et al. Middle-ear disease and schizophrenia: case-control study. Br J Psychiatry 2008; 193: 192-6.

2 Sackett DL, Straus SE, Richardson WS, Rosenberg W, Haynes RB. Evidence Based Medicine. How to Practice and Teach EBM. Churchill Livingstone, 2000.

Ashok K Jainer Coventry \& Warwickshire Partnership Trust, Caludon Centre, Coventry CV2 2TE, UK. Email: ashokjainer@hotmail.com; Supriya M. Shivanandaswamy, Coventry \& Warwickshire Partnership Trust, Caludon Centre, Coventry, UK

doi: 10.1192/bjp.194.1.89a

Author's reply: Jainer \& Shivanandaswamy's comments about the problems of bias in case-control studies are well made. However, our study ${ }^{1}$ was designed to avoid such problems by recruiting all patients with a likely diagnosis of schizophrenia in contact with general practitioners in a defined catchment area. There was no possibility of influencing the selection of individuals 